\title{
Antioxidant Activities of Polysaccharide Fractions Purified from Corn Bran
}

\author{
Linghui Zhu' ${ }^{1}$, Qing $\mathrm{Mo}^{2}{ }^{2}{ }^{,}{ }^{*}$, Jianjun $\mathrm{Ma}^{2}$, Jingjing $\mathrm{Xu}^{2}$ and Miaoli Fang ${ }^{2}$ \\ ${ }^{1}$ Zhejiang Chinese Medical University; Hangzhou, 310053, P. R. China. \\ ${ }^{2}$ Hang Zhou Medical College; Hangzhou, 310053, P. R. China. \\ a Corresponding author: moqing2006@163.com
}

Keywords: Corn bran; Polysaccharide; Composition; Antioxidant activities.

\begin{abstract}
Extractable polysaccharides (CBP) from abundant low-priced by-product of corn bran and three polysaccharide sub-fractions (CBP1, CBP2 and CBP3) were isolated and purified by ion-exchange and DEAE cellulose chromatography. Structural characterization, antioxidant and bile acid binding activities of the polysaccharides were investigated. The average molecular weights (Mw) of CBP1, CBP2 and CBP3 were $342 \mathrm{kDa}, 294 \mathrm{kDa}$ and $186 \mathrm{kDa}$, respectively. Our results showed that CBPs were composed of xylose, glucose, galactose, arabinose and rhamnose, and had typical IR spectra characteristics of polysaccharides. The fraction of CBP3 with the highest protein content $(1.32 \%)$, galactose content $(9.93 \%)$, and the lowest molecular weight $(186 \mathrm{kDa})$ and carbohydrate content $(91.6 \%)$ had the highest antioxidant activities. These findings suggest that isolated polysaccharides from corn bran are potential natural antioxidant that can be used as functional food ingredients.
\end{abstract}

\section{Introduction}

Corn bran which is mostly used as cattle feed is quite abundant, making up one third of the dry weight of annual plants/agricultural crops [1]. Nevertheless, most of the works of polysaccharide extracted from corn bran are just focused on the emulsibility [2]. Recently, polysaccharides from various plants have been recognized as safe and effective antioxidants [3].

Therefore, in this study, the polysaccharides isolated from the crude polysaccharide of corn bran (CBP) were purified by DEAE cellulose chromatography. The results will lay the foundation for further processing of the corn bran, which will underline significant promotions of the agricultural science toward sustainable development.

\section{Materials And Methods}

\subsection{Materials and Chemicals}

Corn bran was collected in Hangzhou, Zhejiang Province, China, and de-oil, de-starched according to Yadav et al. [2]. All other reagents were of analytical grade and used without further purification.

\subsection{Extraction of Crude Polysaccharide}

The crude polysaccharide was extracted on the basis of previous method [2].

\subsection{Isolation and Purification of Polysaccharide}

The above polysaccharide $(1.50 \mathrm{~g})$ was re-dissolved in $50 \mathrm{~mL}$ deionized water, centrifuged at $5000 \mathrm{rpm}$ for $10 \mathrm{~min}$ and then the supernatant was penetrated through a $0.45 \mu \mathrm{m}$ filter. The $5 \mathrm{~mL}$ filtrate was applied to a column of DEAE cellulose chromatography equilibrated with deionized water. And the column was eluted with a linear gradient of $0-1 \mathrm{~mol} / \mathrm{L} \mathrm{NaCl}$ solution at a flow rate of $1 \mathrm{~mL} / \mathrm{min}$ to arrive at homogenous preparation. Three fractions CBP1, CBP2 and CBP3 were obtained, dialyzed and lyophilized for further study, respectively. 


\subsection{Analysis of Carbohydrate and Protein Content}

The carbohydrate and protein content of all the CBP samples obtained above were analyzed by the phenol-sulphuric acid method [4]. Protein was determined by Coomassie Brilliant Blue method [5]. The samples were dissolved to $1 \mathrm{mg} / \mathrm{mL}$ solution and scanned with an ultraviolet spectrophotometer (U-1900UV, Hitachi high-Technologies Corp., Japan).

\subsection{Monosaccharide Composition Analysis}

The sugar composition was analyzed by gas chromatography (Agilent 6890N GC, USA Agilent Technologies) [5].

\subsection{Homogeneity and Molecular Weight Determination}

The homogeneity and molecular weight $(\mathrm{Mw})$ were evaluated and determined by size-exclusion chromatography [6].

\subsection{In vitro Antioxidant Activity Assays}

\subsubsection{Hydroxyl Radical Scavenging Assay}

Hydroxyl radical scavenging activity of each extract solution was measured according to the salicylic method with some modifications [7]. The hydroxyl radical-scavenging ratio was calculated by the following formula:

Scavenging effect $\left.(\%)=\left[1-\left(A_{1}-A_{2}\right) / A_{0}\right)\right] * 100 \%$

Where $\mathrm{A}_{0}$ was the absorbance of the control (without sample), $\mathrm{A}_{1}$ was the result of samples, $\mathrm{A}_{2}$ was the absorbance for background without sodium salicylate.

2.7.2 Superoxide Anion Scavenging Assay

The assay was based on the capacity of the sample to inhibit the photochemical reduction of nitroblue tetrazolium in NADH-NBT-PMS with some modifications [7]. The superoxide anion scavenging ratio was calculated by the following formula:

Scavenging effect $(\%)=\left(1-A_{1} / A_{0}\right) * 100 \%$

Where $A_{0}$ was the absorbance of the control (without sample) and $A_{1}$ was the result of the samples.

Statistical Analysis: The results were means \pm standard deviation (SD) of determinations of triplicate cultures. The data were analyzed by one-way ANOVA and a level of $\mathrm{P}<0.05$ was regarded as statistically significant with Duncan's new multiple ranges.

\section{Result and Discussion}

\subsection{Isolation, Purification and Composition of Polysaccharides}

Table 1. Major Chemical Content and Monosaccharide Components of the Fractions Purified from Corn Bran Polysaccharide.

\begin{tabular}{ccccccccc}
\hline & Carbohydrate & Protein & \multicolumn{5}{c}{ Monosaccharide Composition (mol\%) } \\
\cline { 4 - 8 } & Content (\%) & Content $(\%)$ & Glucose & Galactose & Mannose & Arabinose & Rhamnose & Xylose \\
\hline CBP & 74.7 & $1.50 \%$ & 3.35 & 7.30 & nd & 37.22 & 1.14 & 50.99 \\
CBP1 & 96.3 & $0.57 \%$ & 2.16 & 5.72 & nd & 42.42 & 1.82 & 47.88 \\
CBP2 & 93.4 & $0.92 \%$ & 1.84 & 4.51 & nd & 40.82 & 1.46 & 51.37 \\
CBP3 & 91.6 & $1.32 \%$ & 1.3 & 9.93 & nd & 38.54 & 1.75 & 48.48 \\
\hline
\end{tabular}

nd: Not detected

Table 1 is a summary of carbohydrate, protein content and monosaccharide components of the samples. CBP3 had a lower carbohydrate content of 91.6\% than CBP1 (96.3\%) and CBP2 (93.4\%) but a higher protein content of $1.32 \%$. The samples were composed of five monosaccharides, namely glucose (Glu), galactose (Gal), arabinose (Ara), rhamnose (Rha), xylose (Xyl) with different molar proportions. Arabinose and xylose were the dominant component in all samples. CBP3 had the highest content of galactose (9.93\%), which was over two times than CBP1 and CBP2. The monosaccharide components of the three fractions were not completely the same as those in CBP.

The average molecular weights (Mw) of CBP1, CBP2 and CBP3 were determined as 342, 294, $186 \mathrm{kDa}$ by gel permeation chromatography technique. Weight-average molecular weight and polydispersity ratio were important information because the functional properties of polysaccharide can be influenced greatly by both of them [5]. 


\subsection{Antioxidant Activities of Polysaccharides}

\subsubsection{Hydroxyl Radical-scavenging Activity}

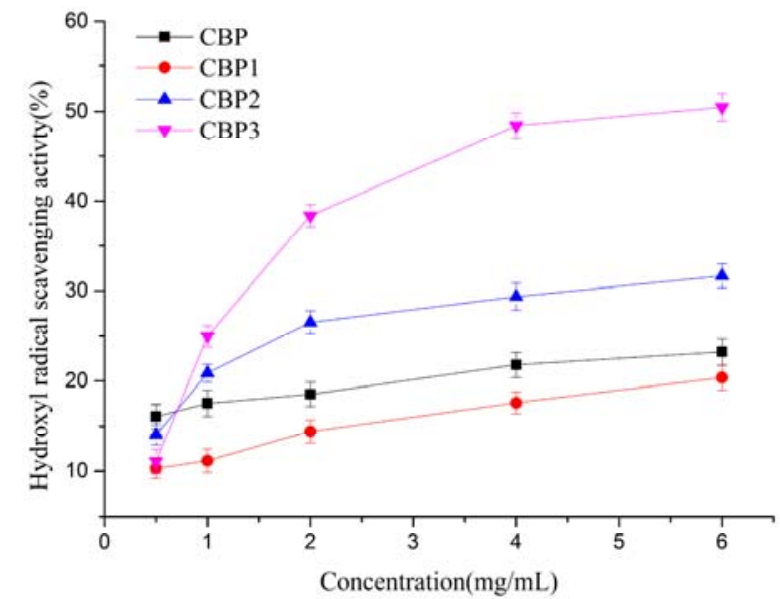

Figure 1. Hydroxyl radical-scavenging rates of CBP, CBP1, CBP2 and CBP3. Each point is the mean \pm SD of triplicates.

As is shown in Fig. 1, the samples exhibited hydroxyl radical-scavenging activity in a dose-dependent manner $(0.5-6.0 \mathrm{mg} / \mathrm{ml})$. CBP3 had a higher activity than CBP1 and CBP2. Unlike CBP2 and CBP3, CBP1 did not show higher scavenging activity than CBP. The scavenging ability of all fractions increased with concentration up to $2.0 \mathrm{mg} / \mathrm{ml}$ and then leveled off. The highest scavenging rates of $\mathrm{CBP}, \mathrm{CBP} 1, \mathrm{CBP} 2$ and $\mathrm{CBP} 3$ were $23.21 \pm 1.47 \%, 20.38 \pm 1.39 \%, 31.64 \pm$ $1.35 \%, 50.47 \pm 1.52 \%$, respectively.

3.2.2 Superoxide Anion-scavenging Activity

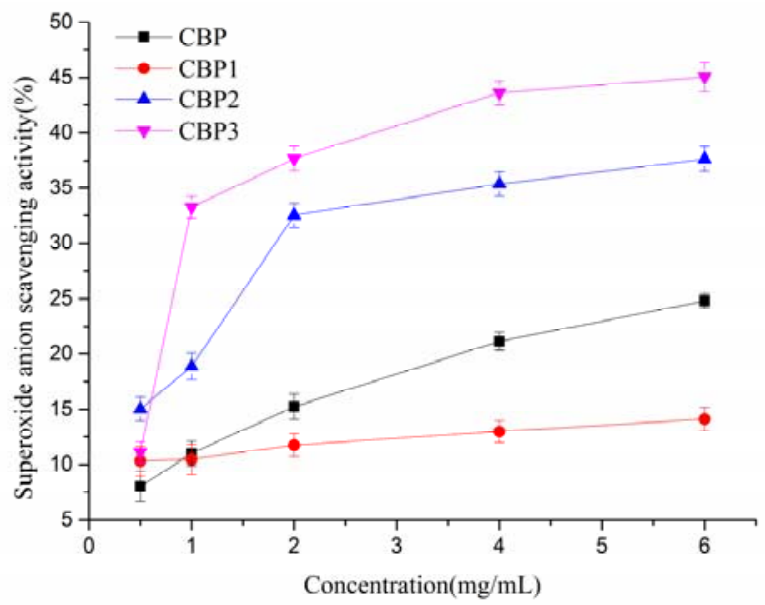

Figure 2. Superoxide anion-scavenging activity of CBP, CBP1, CBP2 and CBP3. Each point is the mean $\pm \mathrm{SD}$ of triplicates.

Fig. 2 shows the superoxide anion-scavenging activity of the CBP and the three fractions. All the samples had scavenging activities in a dose-dependent manner $(0.5-6 \mathrm{mg} / \mathrm{mL})$. The scavenging ability of all fractions increased with concentration up to $2.0 \mathrm{mg} / \mathrm{ml}$ and then leveled off. Like the hydroxyl radical-scavenging activity, CBP1 did not show higher scavenging activity than CBP. The highest scavenging rates of CBP, CBP1, CBP2 and CBP3 were $24.82 \pm 0.64 \%, 14.13 \pm 1.07 \%$, $37.64 \pm 1.09 \%, 45.07 \pm 1.35 \%$, respectively. 


\section{Conclusion}

Corn bran, as a kind of agricultural residues, is abundantly available resource. The fractions had the stronger antioxidant effects and bile acid binding capacity than the native polysaccharide. The changes in protein content, monosaccharide composition, and $\mathrm{Mw} / \mathrm{Mn}$, which may lead to the difference in antioxidant activity. More analysis and evaluation of chemical structure and chain conformations of the $\mathrm{CBP}$ fractions are required for further understanding.

\section{Acknowledgments}

This research was supported by Zhejiang Medical College Foundation (No. 2015XZA03) and Zhejiang Chinese Medical University Foundation (No. 2015ZY30).

\section{References}

[1] S. Kokubun, M. P. Yadav, R. A. Moreau and P. A. Williams, Food Hydrocolloids 41, 164-168 (2014).

[2] M. P. Yadav, R. A. Moreau, A. T. Hotchkiss and K. B. Hicks, Carbohyd. Polym. 87, 1169-1175 (2012).

[3] N. N. Wang, Y. Zhang, X. P. Wang, X. W. Huang, Y. Fei, Y. Yu and D. Shou, Int. J. Biol.Macromol. 83, 103-110 (2016).

[4] M. Dubois, K. A. Gilles, J. K. Hamilton, P. A. Rebers and F. Smith, Anal Chem. 29, 350-356 (1956).

[5] S. C. Li, X. M. Yang, H. L. Ma, J. K. Yan and D. Z. Guo, Carbohyd. Polym. 133, 24-30 (2015).

[6] X. Q. Xu, Y. Hu and L. H. Zhu, J. Taiwan. Inst. Chem. E. 45, 2851-2858 (2014).

[7] C. Y. Huang, S. J. Wu, W. N. Yang, A. W. Kuan and C.Y. Chen, Food Chem. 197, 1121-1129 (2016). 